EXTENDED REPORT

\title{
Alterations in the morphology of lamina cribrosa pores in glaucomatous eyes
}

\author{
G Tezel, K Trinkaus, M B Wax
}

See end of article for authors' affiliations

..................

Correspondence to: Gülgün Tezel, MD, Department of

Ophthalmology and Visual

Sciences, University of

Lovisville School of

Medicine, Kentucky Lions

Eye Center, $301 \mathrm{E}$

Muhammad Ali Blvd,

Lovisville, KY 40202, USA:

gulgun.tezel@louisville.edu

Accepted for publication 5 April 2003

\begin{abstract}
Aims: To determine alterations which occur in the size and shape of lamina cribrosa (LC) pores in glaucomatous eyes over a period of time.

Methods: Baseline and follow up optic disc photographs were retrospectively studied in 39 eyes of 39 patients with glaucoma. Only eyes with a vertical cup to disc ratio equal to or greater than 0.6 were included in the study. In addition, all selected eyes had to have serial optic disc photographs obtained at least 3 years apart allowing clear visualisation of LC surface. The association of the alterations in LC surface morphology with patient specific and eye specific characteristics was statistically analysed.

Results: During a mean study period of 3.90 (SD 0.7) years, individual pore size (mean pore area to disc area ratio) exhibited a significant decrease between baseline and follow up measurements of each eye $(p<0.0001)$. However, during the study period, total pore area to disc area ratio did not change ( $p>0.05)$, and the change in pore shape in some eyes (from circular to more oval and elongated) was statistically insignificant $(p=0.12)$. Although a relation was detectable between the optic disc and lamina cribrosa parameters at a given time, which reflects cumulative effects, during the study period, there was no significant association between the changes of the LC parameters and neural tissue damage. The rate and the magnitude of the changes in individual pore size during the study period were not significantly different among the eyes exhibiting progressive neural rim damage and those staying stable ( $p>0.05)$. Conclusion: These findings demonstrate that the LC surface morphology exhibits changes along with the glaucomatous optic disc damage. However, the clinical appearance of LC surface in glaucomatous eyes may continue to change, even when the neural rim damage is clinically stable. These findings are probably associated with the chronic cellular events of tissue remodelling that occur in the glaucomatous optic nerve head.
\end{abstract}

$\mathrm{O}$ tic disc cupping detected in glaucoma patients by ophthalmoscopy corresponds to characteristic degenerative changes of the optic nerve head $(\mathrm{ONH})$, such as backward bowing and disorganisation of the lamina cribrosa (LC), which accompany the loss of axons. ${ }^{1-5}$ As optic nerve axons exit the eye at the LC, a specialised region of the sclera, they travel through specific openings between the laminar cribriform plates, which are called LC pores. LC pores have been the focus of interest in several clinical and histopathological studies during the past two decades. For example, examination of cross sectioned human ONHs has revealed regional anatomical variations of LC pores. ${ }^{6}$ Mean size of a pore is larger in the superior and inferior parts of the LC in accordance with the greater number of axons travelling through these regions. It has been suggested that larger pores and thinner connective tissue support in the superior and inferior parts of the LC might be associated with the greater susceptibility of nerve fibres passing through these regions. ${ }^{5-9}$

Differences in LC pore shape in glaucomatous eyes have also been associated with increasing severity of the disease. For example, despite the presence of approximately round pores in normal subjects, more elongated pores detected in glaucomatous eyes have been associated with increasing visual field loss. ${ }^{10-12}$ More recently, laser scanning ophthalmoscopy has been introduced for in vivo evaluation of LC morphology, ${ }^{13}{ }^{14}$ and has revealed similar findings. ${ }^{15}$ However, despite all this information, temporal changes of the LC surface morphology in glaucomatous eyes have not been evaluated. Because of interindividual and optic disc size dependent differences in the anatomy of LC, an examination of the alterations of LC pore size and shape in individual patients over a period of time might provide additional information.

Improvements in our understanding of cellular events that occur in the glaucomatous $\mathrm{ONH}^{16-22}$ encouraged us to reexamine the LC surface morphology in glaucomatous eyes over time. Therefore, we studied changes in the morphological features of LC surface in glaucomatous eyes, including the size and shape of pores, using serial optic disc photographs.

\section{PATIENTS AND METHODS}

With a careful review of the records and colour optic disc photographs of patients followed in the glaucoma clinic at Washington University, 39 eyes of 39 patients with glaucoma (21 eyes with primary open angle glaucoma and 18 eyes with normal pressure glaucoma) were selected to be included in this study. Only glaucomatous eyes with a vertical cup to disc ratio equal to or greater than 0.6 were included in this study, because with lesser amounts of optic nerve damage, not enough of the LC surface would be visible for proper evaluation. In addition, only eyes that allowed clear visualisation of the structural details of LC surface by serial photography were included in the study. Even in eyes with no media opacity, if the photographic focus of the serial optic disc photographs did not allow clear visualisation of LC surface details, then those eyes were excluded from the study. Thus, all of the eyes studied met the following inclusion criteria: (1) a vertical cup to disc ratio equal to or greater than $0.6 ;(2)$ the availability of high quality colour stereoscopic optic disc photographs taken at least 3 years apart; and (3) at the time of optic disc photography, the absence of media opacities that impaired clear visualisation 
of LC surface. If both eyes of a patient fulfilled the inclusion criteria (only six of 39 eyes), one randomly selected eye was included in the study.

Inclusion and exclusion criteria for a diagnosis of primary open angle glaucoma or normal pressure glaucoma were previously described. ${ }^{23}{ }^{24}$ Morphometric analysis of digitised optic disc photographs was performed using the NIH Image program (developed at the US National Institutes of Health and available on the internet at rsb.info.nih.gov/nih-image/) in a masked fashion, as previously described. ${ }^{23-26}$ All of the measurements were expressed as a ratio to optic disc size in order to minimise the effects of individual, optic disc size related, age related, and refraction related errors. Parameters evaluated were vertical cup to disc ratio, neural rim area to disc area ratio, number of visible pores, mean pore area to disc area ratio (average of measurements for all visible pores), and total pore area to disc area ratio (total of measurements for all visible pores).

To qualitatively evaluate the shapes of LC pores, they were classified as circular, oval, or striate using the mixed and numbered optic disc photographs to mask the identity and diagnosis of patients. If LC pore shape was not constant through the exposed LC surface, but exhibited regional differences, classification was based on the predominate number of pores with a different shape. LC pore shape was qualitatively evaluated using the entire set of masked optic disc photographs in three separate sequences, and an assessment was made only when at least two of them were constant. A disagreement of all three sequences did not occur in any of the eyes. Some optic disc photographs are presented in figure 1 to illustrate the classification of LC pore shape.

Demographic and clinical data collected from patient charts included age, sex, race, and intraocular pressure (IOP). Mean IOP levels were calculated using all recorded measurements in patient charts before the study period (called baseline IOP), and using all measurements obtained during the study period (called follow up IOP). IOP readings were obtained using applanation tonometry at follow up examinations performed at least yearly. All of the patients were taking antiglaucoma treatment at baseline. We did not study the effects of systemic diseases or specific medical or systemic treatments on LC surface morphology, since they were not constant during the study period or our collection of this information was incomplete.

Reproducibility of the morphometric measurements was determined by calculating the intraexaminer agreement for each LC parameter. After re-evaluating the randomly selected optic disc photographs 10 times, coefficients of variation (range $2.90 \%-4.06 \%$ ) and $95 \%$ confidence intervals were calculated. In respect of both previously determined reproducibility of examiner ${ }^{23}$ and current values, presence of a $5 \%$ difference between baseline and follow up parameters was defined as a change, since it was beyond the $95 \%$ confidence

\begin{tabular}{ll}
$\begin{array}{ll}\text { Table } 1 \text { Baseline patient and eye } \\
\text { characteristics }\end{array}$ \\
\hline Characteristics \\
\hline No & 39 \\
Age (year) & $74.31(7.3)$ \\
Black race & $6(15.4)$ \\
Female sex & $20(51.3)$ \\
Intraocular pressure (mm Hg) & $21.08(2.9)$ \\
Vertical cup to disc ratio & $0.77(0.1)$ \\
Neural rim area to disc area ratio & $0.23(0.1)$ \\
Number of pores & $20.51(2.0)$ \\
Mean pore area to disc area ratio & $0.0055(0.0005)$ \\
Total pore area to disc area ratio & $0.11(0.006)$ \\
Pore shape & $3(7.7)$ \\
Circular & $26(66.7)$ \\
Oval & $10(25.6)$ \\
Striate & \\
\hline *All data are given as the mean (SD) or the number (\%). \\
\hline
\end{tabular}

interval of the intraexaminer reproducibility. Data from all glaucomatous eyes were evaluated together. Differences between baseline and follow up parameters were tested using a paired $t$ test. Relations between the optic disc and LC parameters were examined using Spearman's rank correlation. For categorical variables, Fisher's exact test, and for comparison of continuous variables, the Wilcoxon-MannWhitney and Wilcoxon matched pairs signed rank tests were used. Relations between the change in mean pore area to disc area ratio and other variables were also analysed using a linear logistic regression model. ${ }^{27}$ Odds ratios and 95\% confidence intervals for associations of decreased pore area with patient specific and eye specific variables were obtained. All statistical analyses were carried out using a software, SAS version 8.1. Statistical testing was conducted at the 0.05 level.

\section{RESULTS}

Baseline patient specific and eye specific characteristics are summarised in table 1 . Baseline and follow up IOP were 21.08 (SD 2.9) and 17.00 (2.1) $\mathrm{mm} \mathrm{Hg}$, respectively. Table 2 summarises changes in morphological characteristics over time. During a mean study period of 3.9 (0.7) years (range 35 years), mean pore area to disc area ratio was significantly decreased between baseline and follow up measurements of each eye (paired $t$ test, $\mathrm{p}<0.0001$ ). During the study period, in 19 of the 39 eyes $(49 \%)$, mean pore area to disc area ratio decreased by at least 5\% (range 5.00\%-10.26\% decrease; mean $6.53 \%$ (SD $1.4 \%$ )). This change was beyond the $95 \%$ confidence interval of the reproducibility of the morphometric analysis.

Number of visible pores exhibited an increase (20.51 (2.0) versus $21.76(2.5))$ in 14 eyes $(35.9 \%)$, which were also the eyes with an increase in neural rim damage ( 14 eyes, $35.9 \%$ ).
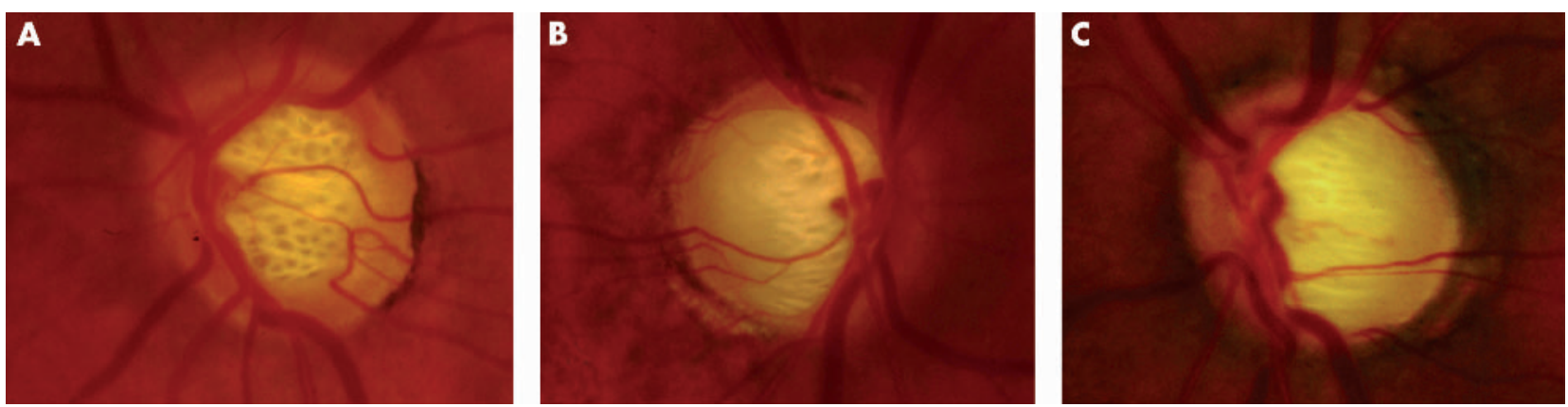

Figure 1 Classification of lamina cribrosa (LC) pore shape. The shapes of the LC pores were qualitatively classified as circular (A), oval (B), or striate $(C)$ 
Table 2 Change in morphological characteristics during follow up*

\begin{tabular}{lcc}
\hline Characteristics & No (\%) & \% change \\
\hline $\begin{array}{l}\text { Vertical cup to disc ratio† } \\
\text { Neural rim area to disc }\end{array}$ & $9(23.1)$ & $6.51(2.0)$ \\
area ratio & $14(35.9)$ & $-24.44(22.5)$ \\
$\begin{array}{l}\text { Number of pores } \\
\text { Mean pore area to disc }\end{array}$ & $19(35.9)$ & \\
$\begin{array}{l}\text { area ratio† } \\
\text { Total pore area to disc }\end{array}$ & 0 & $-6.53(1.4)$ \\
$\begin{array}{l}\text { area ratio† } \\
\text { Pore shape } \\
\quad \text { Circular to oval }\end{array}$ & $1(2.6)$ & \\
$\quad$ Oval to striate & $3(7.7)$ \\
\hline
\end{tabular}

*All data are given as the number (\%) of eyes or mean (SD).

†Data are given if change is at least $5 \%$ during the study period.

Total pore area to disc area ratio did not exhibit a significant change in these eyes during the study period $(0.11) 0.006$ versus $0.11(0.06))$. In addition, as shown in table 2 , some of the eyes exhibited a change in the qualitative classification of pore shape from circular to more oval and elongated, although this was statistically insignificant (Wilcoxon matched pairs signed rank tests, $p=0.12$ ). Figure 2 demonstrates some representative optic disc photographs, taken at baseline and follow up visits, in which LC surface morphology exhibited changes during the study period.

Initially, we sought to determine the relation between morphological parameters of the optic disc and LC at a specific time point, either baseline or follow up. When we first examined their correlation, an increase in vertical cup to disc ratio was found to be strongly correlated with a decrease in neural rim area to disc area ratio (Spearman's rank correlation, $\mathrm{p}<0.0001$ ). This provides an internal control for our observations, since these two parameters are known to be strongly correlated. As shown in table 3, an examination of the correlations between morphological parameters of the optic disc and LC in 39 eyes revealed that mean pore area to disc area ratio decreased and total pore area to disc area ratio increased, along with an increase in vertical cup to disc ratio and a decrease in neural rim area to disc area ratio (Spearman's rank correlation, $\mathrm{p}<0.05$ ).

Similarly, using a univariable linear logistic regression model, a decrease in mean pore area to disc area ratio was found to be associated with increased vertical cup to disc ratio and decreased neural rim area to disc area ratio $(\mathrm{p}=0.01)$. However, there was no significant association between decreased pore area and other parameters studied, which included age, sex, diagnosis, and mean IOP. In addition, we examined whether change in mean pore area to disc area ratio differed between black and white patients. However, confidence intervals for such an association were wide and inconclusive, perhaps largely because of the small number of black patients in our cohort. Analysis of the relations between mean pore area to disc area ratio and different variables in 39 eyes at baseline is shown in table 4 .

Since the univariable analysis did not find any association between mean pore area to disc area ratio and the other variables tested, only covariates for a multivariable analysis were the two optic disc parameters-namely, vertical cup to disc ratio and neural rim area to disc area ratio. However, as a consequence of their close association with each other, only one of the optic disc parameters could be included as a covariate in any given model. Therefore, we considered that such a multivariable model would not be properly conclusive for our data, and would offer little information over that provided by single variable models.

We then sought to determine whether the change in mean pore area to disc area ratio detected during the study period was associated with the change detected over time in the optic disc parameters, including vertical cup to disc ratio and neural rim area to disc area ratio. As given in table 5, increase in the number of visible pores was directly associated with increased neural damage and increased exposure of LC surface, since the eyes exhibiting an increase in pore number were the ones with increased damage to the neural rim (Fisher's exact test, $\mathrm{p}<0.0001$ ). However, there was no significant difference in the rate of change detected over time in individual pore size, or in pore shape, among eyes that either exhibited a progressive neural tissue damage or stayed stable during the study period (Fisher's exact test,
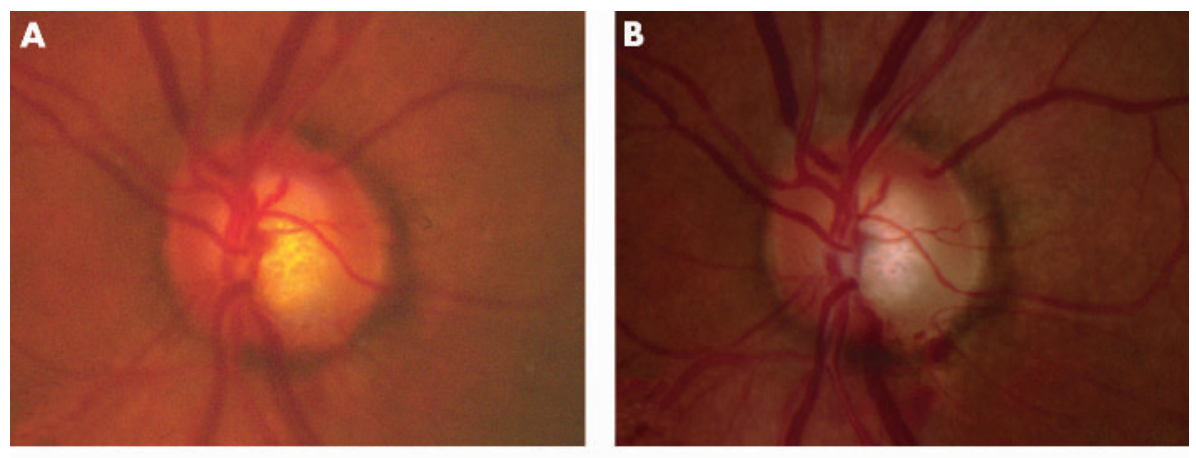

Figure 2 Optic disc photographs of patients with glaucoma. (A) A patient with primary open angle glaucoma at baseline. (B) Follow up of the same eye shown in (A). (C) A patient with normal pressure glaucoma at baseline. (D) Follow up of the same eye shown in (C). Individual size of $\mathrm{LC}$ pores decreased during a follow up period of 5 years.
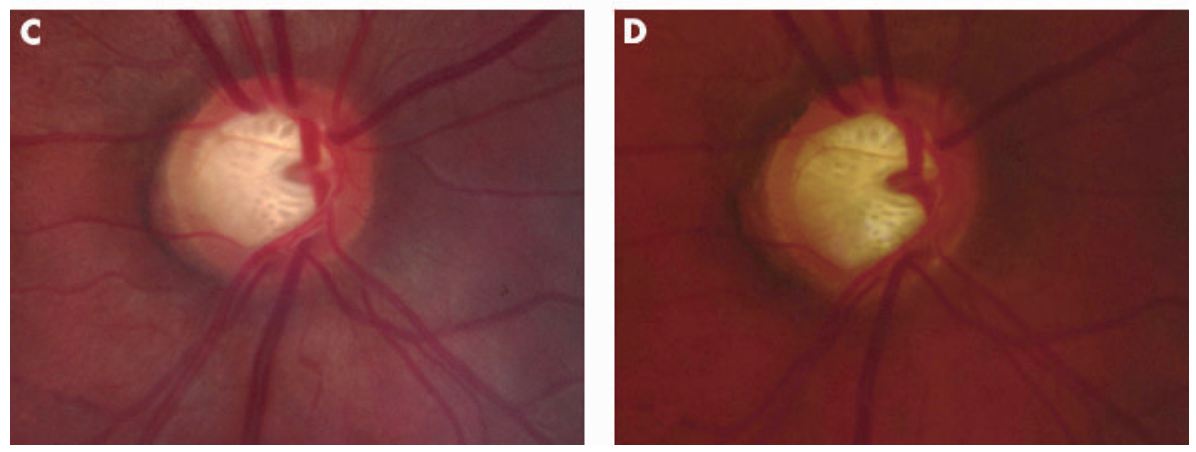
Table 3 Relations between morphological measurements*

\begin{tabular}{|c|c|c|c|}
\hline & $\begin{array}{l}\text { Total pore area to } \\
\text { disc area ratio }\end{array}$ & Vertical cup to disc ratio & $\begin{array}{l}\text { Neural rim area to } \\
\text { disc area ratio }\end{array}$ \\
\hline $\begin{array}{l}\text { Mean pore area to disc } \\
\text { area ratio }\end{array}$ & $-0.0046(0.98)$ & $-0.39(0.017)$ & $0.36(0.025)$ \\
\hline $\begin{array}{l}\text { Total pore area to disc area } \\
\text { ratio }\end{array}$ & & $0.38(0.020)$ & $-0.38(0.018)$ \\
\hline Vertical cup to disc ratio & & & $-1.00(<0.0001)$ \\
\hline
\end{tabular}

$p>0.05)$. These findings demonstrate that decreased pore size was independent from the number of pores, which was used as denominator for the calculation of individual (average) pore size. Whether or not there was a decrease in neural rim area to disc area ratio during the study period, a decrease in mean pore area to disc area ratio was detectable.

In addition to a similar rate of change in mean pore area to disc area ratio over time in eyes with or without a change in neural rim damage, the magnitude of change detected in mean pore area to disc area ratio during the study period was similar between these eyes (Wilcoxon-Mann-Whitney test, $-0.027(0.01)$ versus $-0.022(0.02), p=0.29)$. An examination of the correlation between the change in mean pore area to disc area ratio and the change in vertical cup to disc ratio during the study period similarly revealed that these two variables were not correlated with each other (Spearman's rank correlation, $\mathrm{p}=0.27$ ).

\section{DISCUSSION}

We observed that the individual size of LC pores exhibits a decrease in glaucomatous eyes, while the total area of pores increases along with the glaucomatous damage. A positive correlation was detected between total pore area and glaucomatous optic disc damage, using either baseline or follow up measurements in a cross sectional manner. This was probably related to the fact that the progressive narrowing of neural rim in glaucomatous eyes during the disease period results in increased exposure of LC surface and pores. This is also in accordance with our observation, which demonstrated an increase in the number of visible pores in eyes exhibiting a progression in neural rim damage over time. However, no significant change was detectable in the total area of pores during the study period, which may partly reflect the relatively constant optic disc cupping during the follow up of these treated patients. This observation might also be associated with the significant decrease in individual pore size during the follow up period.

During the follow up period, an elongation in pore shape was detectable only in some of the eyes. This may be the result of the treatment of our patients to reduce IOP, which might have decreased the compression of laminar cribriform plates by mechanical forces created by the elevated IOP. Alternatively, the study period might not have been long enough to detect changes in pore shape in these eyes.

Mechanical forces applied to LC may also account, in part, for the decrease in individual pore size along with the glaucomatous damage. However, although the change in pore shape was insignificant during the study period, the decrease in individual pore size detected in the same cohort was statistically significant. This may suggest that factors, independent of mechanical forces, may also be associated with the decrease in individual pore size detected. Based on recent improvements in our understanding of tissue remodelling, we hypothesise that the decreased pore size may be associated with the chronic cellular events identified in the glaucomatous ONH.

Histopathological studies of the glaucomatous $\mathrm{ONH}$ demonstrate persistent glial activation during the chronic course of glaucomatous neurodegeneration, which is accompanied by an upregulated synthesis of extracellular matrix components. ${ }^{16-20}$ In addition, astrocytes located in the laminar cribriform plates have been noted to migrate into the LC pores. ${ }^{21}$ Recent in vitro studies have demonstrated that the migratory ability of $\mathrm{ONH}$ astrocytes is indeed activated in response to elevated pressure, and may be associated with the characteristic appearance of the glaucomatous $\mathrm{ONH}$, in vivo. ${ }^{22}$ Thus, histopathological and in vitro findings collectively provide evidence that chronically activated $\mathrm{ONH}$ astrocytes in glaucoma migrate into the LC pores $^{21}$ and synthesise extracellular matrix ${ }^{16-20}$ thereby occupying the space within the pores. Our clinical observation of a decrease in individual pore size over time may therefore provide a clinical relevance of these experimental findings.

Although a relation was detectable between the optic disc and LC parameters, cross sectionally, which reflects cumulative effects, during the study period, individual size of pores continued to decrease even when neural rim damage was clinically stable. This observation supports the hypothesis

Table 4 Odds ratio estimates for decrease in pore size (mean pore area to disc area ratio) in a univariable model

\begin{tabular}{llll}
\hline & Odds ratio & $95 \% \mathrm{Cl}$ & $\mathrm{p}$ Value \\
\hline Patient specific factors & & & \\
Age & 1.02 & $(0.94$ to 1.12$)$ & 0.59 \\
Female sex & 0.48 & $(0.14$ to 1.74$)$ & 0.27 \\
Diagnosis of normal pressure glaucoma & 0.73 & $(0.20$ to 2.57$)$ & 0.62 \\
Eye specific factors & & & \\
Mean intraocular pressure & 1.00 & $(0.98$ to 1.02$)$ & 0.86 \\
Vertical cup to disc ratio* & 2.78 & $(1.28$ to 6.00$)$ & 0.01 \\
Neural rim area to disc area ratio* & 0.34 & $(0.15$ to 0.74$)$ & 0.01 \\
Total pore area to disc area ratio† & 0.64 & $(0.20$ to 1.99$)$ & 0.44 \\
\hline
\end{tabular}

*Odds ratio is given for an increase of 0.10 of factor (otherwise for a 1 unit increase). †Odds ratio is given for an increase of 0.01 of factor. 
Table 5 Distribution of change in morphological measurements*

\begin{tabular}{lccc}
\hline & $\begin{array}{l}\text { Decrease in } \\
\text { mean pore } \\
\text { area to disc } \\
\text { area ratio }\end{array}$ & $\begin{array}{l}\text { Change in } \\
\text { pore shape }\end{array}$ & $\begin{array}{c}\text { Increase in } \\
\text { pore number }\end{array}$ \\
\hline $\begin{array}{l}>5 \% \text { change in } \\
\text { neural rim area } \\
\text { to disc area ratio } \\
<5 \% \text { change in }\end{array}$ & $9(23.1)$ & $1(2.6)$ & $14(35.9)$ \\
$\begin{array}{l}\text { neural rim area } \\
\text { to disc area ratio } \\
\begin{array}{l}\text { Statistical } \\
\text { significance (p)t }\end{array}\end{array}$ & 0.19 & $3(7.7)$ & 0 \\
\hline
\end{tabular}

*All data are given as the number (\%) of eyes. tFisher's exact test.

that the remodelling of the glaucomatous $\mathrm{ONH}$ tissue is a chronic series of cellular events. This is also in accordance with the concept that clinical changes in glaucoma occur as a sequel, years after the lowering of elevated IOP. ${ }^{28}$

A previous study by Ogden et al demonstrated that the proportion of the $\mathrm{ONH}$ consisting of pores significantly decreases with age. ${ }^{29}$ This finding was suggested to be associated with the age dependent loss of axons. Since the total area of LC is constant with age, the decreased pore area was thought to be the result of a compensation of neural loss by astroglial tissue with no scar tissue formation. We did not detect any relation between the pore morphology and age, which is probably because of the limited age range in our cohort. Nevertheless, the study of Ogden et al, which signifies contribution of glial cell responses to changes in pore morphology, supports our hypothesis that chronic cellular events identified in the glaucomatous ONH may contribute to decreased pore size detected in these eyes. Selecting eyes with a vertical cup to disc ratio equal to or greater than 0.6 should not cause any artefact related to the decreased pore size over time, since pore size is even larger in areas close to the periphery of the LC.

Age dependent changes in LC morphology detected by Ogden et al were proposed to be associated with increased sensitivity of the elderly to glaucomatous damage. ${ }^{29}$ Contrary to earlier suggestions that a larger pore area is a risk factor for the damage of optic nerve axons, ${ }^{5812}$ these authors have suggested that small pores might lead to the compression of axons as they cross the LC. Similarly, the decreased pore size we detected in glaucomatous eyes over time, which may represent an augmented and accelerated form of age dependent changes in these eyes, may be a factor increasing the vulnerability of injured axons to further biomechanical and/or chemical damage.

Considerable evidence suggests that elevated hydrostatic pressure, in vitro, which simulates elevated IOP, in vivo, can induce the activation of cultured $\mathrm{ONH}$ astrocytes. ${ }^{22}$ 30-32 Therefore, elevated IOP appears to be an important factor associated with continuing tissue remodelling in the glaucomatous $\mathrm{ONH}$, although we did not detect an association between the LC pore morphology and the treated levels of IOP, retrospectively. Whether the further reduction of IOP impedes tissue remodelling, our findings indicate that alterations of LC pore morphology occur even in glaucomatous eyes without clinically detectable neural rim damage. Although it is unclear whether the ONH is the primary site of neural injury in glaucoma, persisting cellular events may alter the clinical features of this region.

Many of the features of our study, including the careful selection of patients, should diminish several vulnerabilities intrinsic to such a retrospective study. Our selection criteria allowed the proper evaluation of LC surface morphology over time using serial optic disc photographs with similar quality. Further clinical studies in larger and prospective patient cohorts and further experimental studies can facilitate a better understanding of how the characteristic optic disc appearance develops in glaucoma, and what damaging effects on neural tissue are present at the $\mathrm{ONH}$.

\section{ACKNOWLEDGEMENTS}

This study was supported in part by the Glaucoma Foundation, New York, NY, USA; the Glaucoma Research Foundation, San Francisco, CA, USA; and the American Health Assistance Foundation, Clarksburg, MD, USA. The authors thank Dr Michael A Kass (Washington University, St Louis, MO, USA) for providing clinical data and for valuable comments on the manuscript.

\section{Authors' affiliations}

G Tezel, Department of Ophthalmology and Visual Sciences, University of Louisville School of Medicine, Louisville, KY, USA

K Trinkaus, Division of Biostatistics, Washington University School of Medicine, St Louis, MO, USA

M B Wax, Alcon Laboratories Inc, Fort Worth, TX and Department of Ophthalmology of Texas Southwestern Medical School, Dallas TX, USA

The authors have no proprietary interest in any of the materials used in this study.

\section{REFERENCES}

1 Anderson DR. Pathology of the glaucomas. Br J Ophthalmol 1972;56:146-56

2 Quigley HA, Green WR. The histology of human glaucoma cupping and optic nerve damage: clinicopathologic correlation in 21 eyes. Ophthalmology 1979;86:1803-30.

3 Minckler DS, Spaeth GL. Optic nerve damage in glaucoma. Surv Ophthalmol 1981;26:128-48

4 Quigley HA, Addicks EM, Green WR, et al. Optic nerve damage in human glaucoma. II. The site of injury and susceptibility to damage. Arch Ophthalmol 1981;99:635-49.

5 Quigley HA, Hohman RM, Addicks EM, et al. Morphologic changes in the lamina cribrosa correlated with neural loss in open-angle glaucoma. Am J Ophthalmol 1983;95:673-91.

6 Radius RL, Gonzales M. Anatomy of the lamina cribrosa in human eyes. Arch Ophthalmol 1981;99:2159-62.

7 Quigley HA, Addicks EM. Regional differences in the structure of the lamina cribrosa and their relation to glaucomatous optic nerve damage. Arch Ophthalmol 1981;99:137-43.

8 Dandona L, Quigley HA, Brown AE, et al. Quantitative regional structure of the normal human lamina cribrosa. A racial comparison. Arch Ophthalmol 1990; 108:393-8

9 Jonas JB, Mardin CY, Schlotzer-Schrehardt U, et al. Morphometry of the human lamina cribrosa surface. Invest Ophthalmol Vis Sci 1991:32:401-5.

10 Susanna $\mathbf{R}$. The lamina cribrosa and visual field defects in open-angle glaucoma. Can J Ophthalmol 1983;18:124-6.

11 Miller KM, Quigley HA. Comparison of optic disc features in low-tension and typical open-angle glaucoma. Ophthalmic Surg 1987;18:882-9.

12 Miller KM, Quigley HA. The clinical appearance of the lamina cribrosa as a function of the extent of glaucomatous optic nerve damage. Ophthalmology 1988;95:135-8.

13 Bhandari A, Fontana L, Fitzke FW, et al. Quantitative analysis of the lamina cribrosa in vivo using a scanning laser ophthalmoscope. Curr Eye Res 1997; 16:1-8.

14 Maeda H, Nakamura M, Yamamoto M. Morphometric features of laminar pores in lamina cribrosa observed by scanning laser ophthalmoscopy. Jpn J Ophthalmol 1999;43:415-21.

15 Fontana L, Bhandari A, Fitzke FW, et al. In vivo morphometry of the lamina cribrosa and its relation to visual field loss in glaucoma. Curr Eye Res 1998;17:363-9.

16 Hernandez MR, Andrzejewska WM, Neufeld AH. Changes in the extracellular matrix of the human optic nerve head in primary open-angle glaucoma. Am J Ophthalmol 1990;109:180-8.

17 Morrison JC, Dorman-Pease ME, Dunkelberger GR, et al. Optic nerve head extracellular matrix in primary optic atrophy and experimental glaucoma. Arch Ophthalmol 1990;108:1020-4.

18 Quigley HA, Dorman-Pease ME, Brown AE. Quantitative study of collagen and elastin of the optic nerve head and sclera in human and experimental monkey glaucoma. Curr Eye Res 1991;10:877-88.

19 Varela HJ, Hernandez MR. Astrocyte responses in human optic nerve head with primary open-angle glaucoma. J Glaucoma 1997;6:303-13.

20 Ricard CS, Pena JD, Hernandez MR. Differential expression of neural cell adhesion molecule isoforms in normal and glaucomatous human optic nerve heads. Brain Res Mol Brain Res 1999;74:69-82. 
21 Hernandez MR. The optic nerve head in glaucoma: role of astrocytes in tissue remodeling. Prog Retin Eye Res 2000;19:297-321.

22 Tezel G, Hernandez MR, Wax MB. In vitro evaluation of reactive astrocyte migration, a component of tissue remodeling in glaucomatous optic nerve head. Glia 2001;34:178-89.

23 Tezel G, Kass MA, Kolker AE, et al. Comparative optic disc analysis in normal pressure glaucoma, primary open-angle glaucoma, and ocular hypertension. Ophthalmology 1996;103:2105-13.

24 Tezel G, Siegmund KD, Trinkaus K, et al. Clinical factors associated with progression of glaucomatous optic disc damage in treated patients. Arch Ophthalmol 2001;119:813-18

25 Tezel G, Kolker AE, Kass MA, et al. Parapapillary chorioretinal atrophy in patients with ocular hypertension. I. An evaluation as a predictive factor for the development of glaucomatous damage. Arch Ophthalmol 1997; 115:1503-8.

26 Tezel G, Dorr D, Kolker AE, Wax MB, et al. Concordance of parapapillary chorioretinal atrophy in ocular hypertension with visual field defects that accompany glaucoma development. Ophthalmology 2000; 107:11194-9.

27 Hosmer D, Lemeshow S. Applied logistic regression. New York: Wiley, 1989.

28 Brubaker RF. Delayed functional loss in glaucoma. LII Edward Jackson Memorial Lecture. Am J Ophthalmol 1996;121:473-83.

29 Ogden TE, Duggan J, Danley K, et al. Morphometry of nerve fiber bundle pores in the optic nerve head of the human. Exp Eye Res 1988;46:559-68

30 Hernandez MR, Pena JDO, Selvidge JA, et al. Hydrostatic pressure stimulates synthesis of elastin in cultured optic nerve head astrocytes. Glia (in press).

31 Ricard CS, Kobayashi S, Pena JD, et al. Selective expression of neural cell adhesion molecule (NCAM)-180 in optic nerve head astrocytes exposed to elevated hydrostatic pressure in vitro. Brain Res Mol Brain Res 2000;81:62-79.

32 Agapova OA, Ricard CS, Salvador-Silva M, et al. Expression of matrix metalloproteinases and tissue inhibitors of metalloproteinases in human optic nerve head astrocytes. Glia 2001;33:205-16.

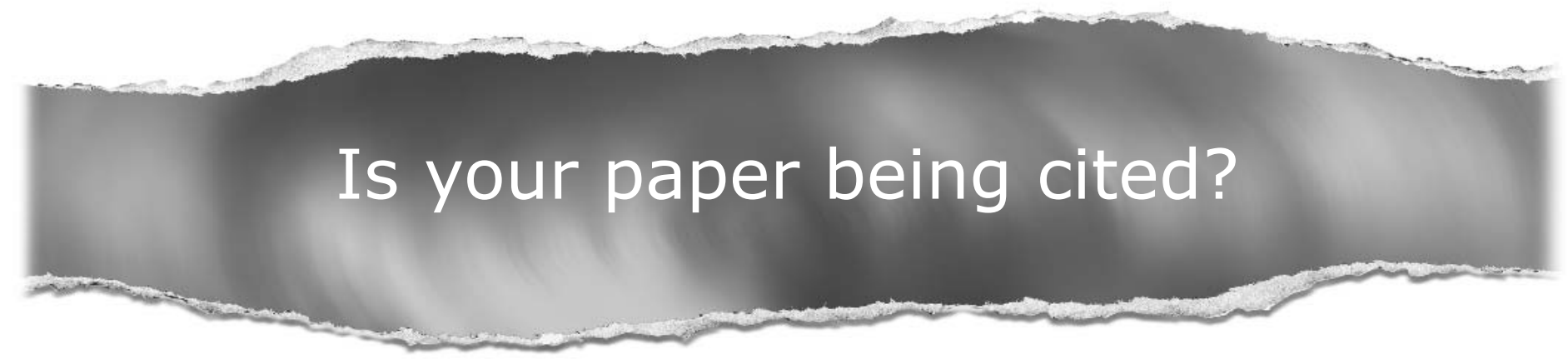

\section{CiteTrack service}

CiteTrack will alert you by email whenever new content in the British Journal of Ophthalmology or a participating journal is published that matches criteria you want to track

Topics: Tell CiteTrack which words or subjects to watch for in new content Authors: Be alerted whenever key authors you are following publish a new paper Articles: Know whenever a paper of interest to you is referenced by another paper

\section{www.bjophthalmol.com}

